\title{
CHALCONSEMICARBAZONE: A NEW SCAFFOLD FOR ANTIEPILEPTIC DRUG DISCOVERY
}

\author{
HEMENDRA PRATAP SINGH ${ }^{a}$, C S CHAUHAN ${ }^{a}, S N$ PANDEYA ${ }^{b}$, CHANDRA SHEKHAR SHARMA , \\ ${ }^{a} B$ N College of Pharmacy, Udaipur, Rajasthan, India \\ ${ }^{b}$ Saroj Institute of Pharmacy, Lucknow, UP, India \\ (Received: May 13, 2009 - Accepted: January 12, 2010)
}

\begin{abstract}
During our investigation in the area of epileptic drug discovery, we have identified that the available conventional antiepileptic drugs are effective in 60 $80 \%$ patients and in specific type of seizures and having various undesirable side effects. But in present time a new class aryl semicarbazone is emerged as new pharmacophore in epileptic drug discovery having broad spectrum activity. On the bases of work done in this area we have applied hybridization of pharmacophore strategy of drug design and developed a new pharmacophore. We have also designed a scheme for synthesizing such pharmacophore and performed their pharmacological screening for the protection of seizures, behavioral study and CNS activity. The compound 1-[1-(2,4-dihydroxyphenyl)-3-(2-hydroxyphenyl) allylidene]-4-(2-fluorophenyl) semicarbazide (8) emerged as the most active prototype molecule in all the models.
\end{abstract}

Key words: semicarbazide, actophotometer, antiepileptic

\section{INTRODUCTION}

Epilepsy is a collective term that includes over 40 different types of human seizure disorders. Approximately $1 \%$ of the world population at any one time ( $>50$ million people worldwide) is afflicted with this serious neurological disorder. Although the current drugs provide adequate seizure control in many patients, it is roughly estimated that up to $28-30 \%$ of patients are poorly treated with the available antiepileptic drugs (AEDs). Moreover, many AEDs have serious side effects and lifelong medication may be required. Hence, with all of these factors in mind, it has been suggested that the focus of epilepsy research should be directed to identifying the underlying mechanism of epileptogenesis and the subsequent "expression" of seizure activity, rather than resorting primarily to symptom control, that is, mere suppression of seizures [1-4].

In the past decade, various aryl semicarbazones have been designed that were structurally dissimilar from many common anticonvulsants containing the dicarboximide function (CONRCO), which may contribute to toxic side effects. Consistent advances in the design of novel anticonvulsant agents have been obtained through the works of Dimmock and his collegues, which included various aryl semicarbazones and (aryloxy) aryl semicarbazones. The terminal primary amino group was implicated in hydrogen bonding. Using the semicarbazone template, Pandeya and co-workers demonstrated through a series of successive works the significant anticonvulsant potential in animal epilepsy models for the N4-(substituted phenyl) semicarbazones [5-17].

So, on the bases of the work done in this area, in our present research work we have used the hybridization of pharmacophore technique of drug design and fused both the nucleus to form a novel pharmacophore 'chalconesemicarbazone'. We have also designed a synthetic scheme to synthesize this pharmacophore moiety and its derivatives and done pharmacological screening.

\section{Experimental Section}

Chemistry

All the compounds were synthesized according to the given synthetic scheme figure 1. Melting points were measured in open capillary tubes on a Büchi 530 melting point apparatus and were uncorrected. Infrared (IR) and proton nuclear magnetic resonance $\left({ }^{1} \mathrm{H}\right.$ NMR) spectra were recorded for the compounds on Jasco IR Report $100(\mathrm{KBr})$ and Brucker Advance $(300 \mathrm{MHz})$ instruments, respectively. Chemical shifts are reported in parts per million (ppm) using tetramethylsilane (TMS) as an internal standard. All exchangeable protons were confirmed by addition of $\mathrm{D}_{2} \mathrm{O}$. Mass spectra were measured with a Shimadzu GC-MS-QP5000 spectrophotometer. Only molecular ions (M+) and base peaks are given. Elemental analyses $(\mathrm{C}, \mathrm{H}$ and $\mathrm{N})$ were undertaken with a Perkin-Elmer model $240 \mathrm{C}$ analyzer, and all analyses were consistent with theoretical values (within $(0.4 \%)$ ). The homogeneity of the compounds was monitored by ascending thin-layer chromatography (TLC) on silica gel $\mathrm{G}$ (Merck) coated aluminum plates, visualized by iodine vapor and UV light.

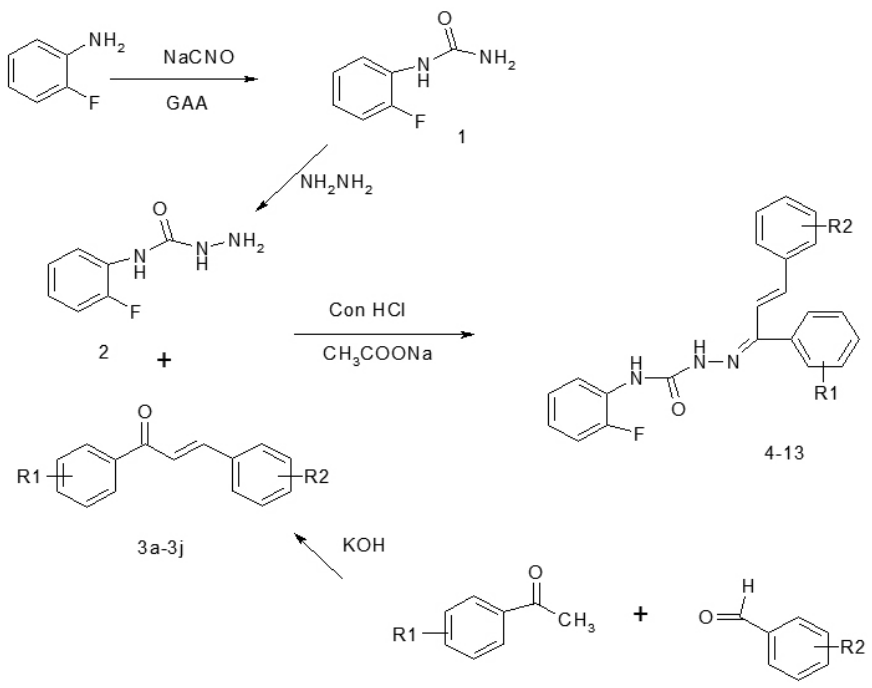

Figure 1: scheme for the synthesis of 2-flourophenyl chalconylsemicarbazones.

Synthesis of substituted chalcone derivatives

Substituted benzaldehydes $(0.012 \mathrm{~mol})$ were added to a mixture of substituted acetophenones $(0.01 \mathrm{~mol})$ in $25 \mathrm{ml}$ of ethanol in a $200 \mathrm{ml}$ beaker. The content of the beaker was mixed well and to that $10 \mathrm{ml}$ of $10 \%$ potassium hydroxide solution was added and stirred vigorously at $25^{\circ} \mathrm{C}$ until the mixture was so thick that stirring was no longer effective $(3-4 \mathrm{~h})$. After the completion of the stirring, the reaction mixture was kept in a refrigerator overnight. The reaction mixture was then diluted with ice-cold water $(50 \mathrm{ml})$, acidified with $10 \%$ aqueous hydrochloric acid to precipitate the chalcones. The product was filtered with suction on a Büchner funnel, washed with cold water until the washings were neutral to litmus and then washed with $10 \mathrm{ml}$ of ice-cold rectified spirit. The dried products were recrystallized from chloroform. The physicochemical parameters of the synthesized chalcone derivatives were given in table 1.

Compounds $3 \mathbf{a}-\mathbf{j}$ (Table 1) gave positive test for chalcone and positive ferric chloride test.)

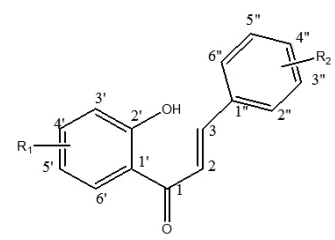


Table 1: structure and physicochemical properties of chalcone derivatives.

\begin{tabular}{|c|c|c|c|c|c|c|}
\hline com & $\mathbf{R}_{1}$ & $\mathbf{R}_{2}$ & Mol for & $\begin{array}{l}\text { Mp } \\
\left({ }^{\circ} \mathbf{C}\right)\end{array}$ & $\begin{array}{c}\text { yield } \\
(\%)\end{array}$ & $\begin{array}{c}R_{\mathrm{f}} \\
\text { value }\end{array}$ \\
\hline $3 a$ & $\mathrm{H}$ & $\mathrm{H}$ & $\mathrm{C}_{15} \mathrm{H}_{12} \mathrm{O}_{2}$ & 89 & 85 & 0.80 \\
\hline $3 b$ & $\mathrm{H}$ & 4"-OH & $\mathrm{C}_{15} \mathrm{H}_{12} \mathrm{O}_{3}$ & 164 & 85 & 0.83 \\
\hline $3 \mathrm{c}$ & $\mathrm{H}$ & 4"-OCH3 & $\mathrm{C}_{16} \mathrm{H}_{14} \mathrm{O}_{3}$ & 135 & 85 & 0.82 \\
\hline $3 d$ & $\mathrm{H}$ & $\begin{array}{c}4 "- \\
\mathrm{N}(\mathrm{CH} 3) 2\end{array}$ & $\mathrm{C}_{17} \mathrm{H}_{17} \mathrm{NO}_{2}$ & 155 & 85 & 0.78 \\
\hline $3 \mathrm{e}$ & $\begin{array}{l}4^{\prime}- \\
\mathrm{OH}\end{array}$ & 6"-OH & $\mathrm{C}_{15} \mathrm{H}_{12} \mathrm{O}_{4}$ & 216 & 90 & 0.85 \\
\hline $3 f$ & $\begin{array}{l}4^{\prime}- \\
\mathrm{OH}\end{array}$ & $\begin{array}{c}4 "- \\
\mathrm{N}(\mathrm{CH} 3) 2\end{array}$ & $\mathrm{C}_{17} \mathrm{H}_{17} \mathrm{NO}_{3}$ & 174 & 90 & 0.81 \\
\hline $3 \mathrm{~g}$ & $\mathrm{H}$ & 6"-OH & $\mathrm{C}_{15} \mathrm{H}_{12} \mathrm{O}_{3}$ & 166 & 85 & 0.86 \\
\hline $3 \mathrm{~h}$ & $5^{\prime} \mathrm{OH}$ & 6"-OH & $\mathrm{C}_{15} \mathrm{H}_{12} \mathrm{O}_{4}$ & 218 & 85 & 0.84 \\
\hline $3 \mathrm{i}$ & $5^{\prime} \mathrm{OH}$ & 4"-OH & $\mathrm{C}_{15} \mathrm{H}_{12} \mathrm{O}_{4}$ & 208 & 85 & 0.87 \\
\hline $3 \mathrm{j}$ & $5^{\prime} \mathrm{OH}$ & 4"-OCH3 & $\mathrm{C}_{16} \mathrm{H}_{14} \mathrm{O}_{4}$ & 152 & 85 & 0.79 \\
\hline
\end{tabular}

\section{Spectral analysis of some selected compounds:}

(3a) ${ }^{1} \mathrm{H}-\mathrm{NMR}\left(\delta / \mathrm{ppm}\right.$ in $\left.\mathrm{CDCl}_{3}\right): 5.0\left(\mathrm{~s}, 1 \mathrm{H}, 2^{\prime}-\mathrm{OH}\right), 7.14$ (dd, $J=7.9$, $1.8 \mathrm{~Hz}, 1 \mathrm{H}, 4$ " - $\mathrm{H}), 7.21$ (d, $J=7.9 \mathrm{~Hz}, 2 \mathrm{H}, 3$ ", $5-\mathrm{H}$ "), 7.30 (d, $J=7.9 \mathrm{~Hz}$, $2 \mathrm{H}, 2 ", 6$ " - $\mathrm{H}), 7.56$ (s, 1H, $-\mathrm{CH}=\mathrm{CH}-), 7.64$ (m, $J=8.3 \mathrm{~Hz}, 4 \mathrm{H}, \mathrm{Ar}-\mathrm{H}), 7.90$ (s, $1 \mathrm{H},-\mathrm{CH}=\mathrm{CH}-)$. IR $\left(\mathrm{KBr} / \mathrm{cm}^{-1}\right): 3480(-\mathrm{OH}), 1748-1716(-\mathrm{CO}), 1670(-$ $\mathrm{CH}=\mathrm{CH}-$ ) , 1616, 1558 (aromatic), 754, 697 (monosubstituted benzene).

(3b) ' ${ }^{\mathrm{H}-\mathrm{NMR}}\left(\mathrm{\delta} / \mathrm{ppm}\right.$ in $\left.\mathrm{CDCl}_{3}\right): 5.0(\mathrm{~s}, 1 \mathrm{H}, 2$ ' $-\mathrm{OH}), 5.1(\mathrm{~s}, 1 \mathrm{H}, 4$ '" $-\mathrm{OH}), 6.68(\mathrm{~d}, J=7.9 \mathrm{~Hz}, 2 \mathrm{H}, 3 ", 5$ " $-\mathrm{H}), 7.13(\mathrm{~d}, J=8.0 \mathrm{~Hz}, 2 \mathrm{H}, 2 ", 6 "-\mathrm{H})$, $7.64-6.92(\mathrm{~m}, J=8.3 \mathrm{~Hz}, 4 \mathrm{H}, \mathrm{Ar}-\mathrm{H}), 7.56(\mathrm{~s}, 1 \mathrm{H},-\mathrm{CH}=\mathrm{CH}-), 7.90(\mathrm{~s}, 1 \mathrm{H}$, $-\mathrm{CH}=\mathrm{CH}-), \mathrm{IR}\left(\mathrm{KBr} / \mathrm{cm}^{-1}\right)$ : 3480, $3345(-\mathrm{OH}), 1771,1732(-\mathrm{CO}), 1682(-$ $\mathrm{CH}=\mathrm{CH}-$ ), 1603, 1575 (aromatic), 834 (p-disubstituted benzene).

(3c) ' $\mathrm{H}-\mathrm{NMR}\left(\delta / \mathrm{ppm}\right.$ in $\left.\mathrm{CDCl}_{3}\right): 3.73\left(\mathrm{~s}, 3 \mathrm{H}, 4\right.$ " $\left.-\mathrm{OCH}_{3}\right), 5.0(\mathrm{~s}, 1 \mathrm{H}, 2$ ' $-\mathrm{OH}), 6.72(\mathrm{~d}, J=7.9 \mathrm{~Hz}, 2 \mathrm{H}, 3$ " , 5" -H), 7.19 (d, $J=7.9 \mathrm{~Hz}, 2 \mathrm{H}, 2$ " , 6" -H), $7.56(\mathrm{~s}, 1 \mathrm{H},-\mathrm{CH}=\mathrm{CH}-), 7.64-6.92(\mathrm{~m}, J=8.1 \mathrm{~Hz}, 4 \mathrm{H}, \mathrm{Ar}-\mathrm{H}), 7.90(\mathrm{~s}, 1 \mathrm{H}$, $-\mathrm{CH}=\mathrm{CH}-), \mathrm{IR}\left(\mathrm{KBr} / \mathrm{cm}^{-1}\right): 3480,3446(-\mathrm{OH}), 1748,1716(-\mathrm{CO}), 1670(-$ $\mathrm{CH}=\mathrm{CH}-), 1605,1575$ (aromatic), 834 (p-disubstituted benzene).

(3d) ${ }^{1} \mathrm{H}-\mathrm{NMR}\left(\delta / \mathrm{ppm}\right.$ in $\left.\mathrm{CDCl}_{3}\right): 2.8\left(\mathrm{~s}, 6 \mathrm{H}, 4{ }^{\prime}{ }^{-}-\mathrm{NMe}_{2}\right), 5.0(\mathrm{~s}, 1 \mathrm{H}, 2$ ' $-\mathrm{OH}), 6.54$ (d, $J=7.9 \mathrm{~Hz}, 2 \mathrm{H}, 3$ " , 5" -H), 7.12 (d, $J=8.0 \mathrm{~Hz}, 2 \mathrm{H}, 2$ ", 6" - H), $7.56(\mathrm{~s}, 1 \mathrm{H},-\mathrm{CH}=\mathrm{CH}-), 7.64-6.92(\mathrm{~m}, J=7.9 \mathrm{~Hz}, 4 \mathrm{H}, \mathrm{Ar}-\mathrm{H}), 7.90(\mathrm{~s}, 1 \mathrm{H}$, $\left.{ }_{-} \mathrm{CH}=\mathrm{CH}-\right), \mathrm{IR}\left(\mathrm{KBr} / \mathrm{cm}^{-1}\right): 3480,3446(-\mathrm{OH}), 1748,1716(-\mathrm{CO}), 1670(-$ $\mathrm{CH}=\mathrm{CH}-), 1621,1558,1521$ (aromatic), 1312 (C-N stretching in Ar amines), 835 (p-disubstituted ben-zene).

Please to correct the NMR data as above.

(3e) ${ }^{1} \mathrm{H}-\mathrm{NMR}\left(\delta / \mathrm{ppm}\right.$ in $\left.\mathrm{CDCl}_{3}\right): 5.0(\mathrm{~s}, 3 \mathrm{H}, 2$ ' , 4', 6" $-\mathrm{OH}), 6.68(\mathrm{~d}$, $J=7.9 \mathrm{~Hz}, 2 \mathrm{H}, 3$ " , 5" -H), $7.13(\mathrm{~d}, J=7.9 \mathrm{~Hz}, 2 \mathrm{H}, 2$, 4 " $-\mathrm{H}), 7.39$ (s, $1 \mathrm{H},-$ $\mathrm{CH}=\mathrm{CH}-), 7.47-6.39(\mathrm{~m}, J=8.2 \mathrm{~Hz}, 3 \mathrm{H}, \mathrm{Ar}-\mathrm{H}), 8.17(\mathrm{~s}, 1 \mathrm{H},-\mathrm{CH}=\mathrm{CH}-)$, IR $\left(\mathrm{KBr} / \mathrm{cm}^{-1}\right): 3841(-\mathrm{OH}), 1732,1698(-\mathrm{CO}), 1670(-\mathrm{CH}=\mathrm{CH}-), 1616,1558$ (aromatic), 727, 652 (monosubstituted benzene).

(3f) ${ }^{1} \mathrm{H}-\mathrm{NMR}\left(\delta / \mathrm{ppm}\right.$ in $\left.\mathrm{CDCl}_{3}\right): 2.85$ (s, 6H, 4" $\left.-\mathrm{NMe}_{2}\right), 5.0$ (s, 2H, 2' , 4' - OH), 6.54 (d, $J=7.9 \mathrm{~Hz}, 2 \mathrm{H}, 3$ " , 5 " - $\mathrm{H}), 7.12$ (d, $J=7.9 \mathrm{~Hz}, 2 \mathrm{H}, 2$ ", 6 " $-\mathrm{H}), 7.56(\mathrm{~s}, 1 \mathrm{H},-\mathrm{CH}=\mathrm{CH}-), 7.47-6.39(\mathrm{~m}, J=8.1 \mathrm{~Hz}, 3 \mathrm{H}, \mathrm{Ar}-\mathrm{H}), 7.90(\mathrm{~s}$, $1 \mathrm{H},-\mathrm{CH}=\mathrm{CH}-)$, IR $\left(\mathrm{KBr} / \mathrm{cm}^{-1}\right): 3480(-\mathrm{OH}), 1748,1697(-\mathrm{CO}), 1670(-$ $\mathrm{CH}=\mathrm{CH}-$ ) 1616,1540 (aromatic), 1316 (C-N stretching in Ar. amines), 824 (p-disubstituted benzene).

(3g) ${ }^{1} \mathrm{H}-\mathrm{NMR}\left(\delta / \mathrm{ppm}\right.$ in $\left.\mathrm{CDCl}_{3}\right): 5.0(\mathrm{~s}, 2 \mathrm{H}, 2$ ', 6 ' $-\mathrm{OH}), 7.11-6.75(\mathrm{~m}$, $J=8.2 \mathrm{~Hz}, 4 \mathrm{H}, \mathrm{Ar}-\mathrm{H}), 7.14(\mathrm{dd}, J=7.9,1.8 \mathrm{~Hz}, 1 \mathrm{H}, 4 "-\mathrm{H}), 7.21(\mathrm{~d}, J=7.9 \mathrm{~Hz}$, $2 \mathrm{H}, 3$ " , 5" $-\mathrm{H}), 7.30(\mathrm{~s}, 1 \mathrm{H}, 2$ " $-\mathrm{H}), 7.56(\mathrm{~s}, 1 \mathrm{H},-\mathrm{CH}=\mathrm{CH}-), 7.90(\mathrm{~s}, 1 \mathrm{H}$, $-\mathrm{CH}=\mathrm{CH}-), \mathrm{IR}\left(\mathrm{KBr} / \mathrm{cm}^{-1}\right)$ : 3391, $3209(-\mathrm{OH}), 1748,1698(-\mathrm{CO}), 1653(-$ $\mathrm{CH}=\mathrm{CH}-$ ) , 1623, 1576 (aromatic), 728, 697 (monosubstituted benzene)s.

(3h) ${ }^{1} \mathrm{H}-\mathrm{NMR}\left(\delta / \mathrm{ppm}\right.$ in $\left.\mathrm{CDCl}_{3}\right): 5.0$ (s, 3H, 2', 5', 6" -OH), 6.68 (d, $\left.J=7.9 \mathrm{~Hz}, 1 \mathrm{H}, 3^{\prime}-\mathrm{H}\right), 6.77\left(\mathrm{dd}, J=7.9,1.8 \mathrm{~Hz}, 1 \mathrm{H}, 6^{\prime}-\mathrm{H}\right), 6.97(\mathrm{dd}, J=7.9,1.8 \mathrm{~Hz}$, $\left.1 \mathrm{H}, 4^{\prime}-\mathrm{H}\right), 7.11-6.75(\mathrm{~m}, J=8.3 \mathrm{~Hz}, 4 \mathrm{H}, \mathrm{Ar}-\mathrm{H}), 7.39(\mathrm{~s}, 1 \mathrm{H},-\mathrm{CH}=\mathrm{CH}-)$, 8.17 (s, $1 \mathrm{H},-\mathrm{CH}=\mathrm{CH}-)$, IR $\left(\mathrm{KBr} / \mathrm{cm}^{-1}\right): 3446(-\mathrm{OH}), 1748,1698(-\mathrm{CO})$, $1670,1652(-\mathrm{CH}=\mathrm{CH}-), 1616,1540$ (aromatic), 714, 673 (monosubstituted benzene).

(3i) ' $\mathrm{H}-\mathrm{NMR}\left(\delta / \mathrm{ppm}\right.$ in $\left.\mathrm{CDCl}_{3}\right): 5.0$ (s, 3H, 2', 5', 4” $\left.-\mathrm{OH}\right), 6.68(\mathrm{~d}$, $J=7.9 \mathrm{~Hz}, 2 \mathrm{H}, 3 ", 5 "-\mathrm{H}), 7.11-6.75(\mathrm{~m}, J=8.3 \mathrm{~Hz}, 3 \mathrm{H}, \mathrm{Ar}-\mathrm{H}), 7.13$ (d, $J=7.9 \mathrm{~Hz}, 2 \mathrm{H}, 2 ", 6 "$ " $\mathrm{H}), 7.56(\mathrm{~s}, 1 \mathrm{H},-\mathrm{CH}=\mathrm{CH}-), 7.90(\mathrm{~s}, 1 \mathrm{H},-\mathrm{CH}=\mathrm{CH}-)$, IR $\left(\mathrm{KBr} / \mathrm{cm}^{-1}\right): 3244(-\mathrm{OH}), 1732,1698(-\mathrm{CO}), 1683(-\mathrm{CH}=\mathrm{CH}-), 1646,1557$ (aromatic), 834 (p-disubstituted benzene).

(3j) ' $\mathrm{H}-\mathrm{NMR}\left(\delta / \mathrm{ppm}\right.$ in $\left.\mathrm{CDCl}_{3}\right): 3.73\left(\mathrm{~s}, 3 \mathrm{H}, 4\right.$ "' $\left.-\mathrm{OCH}_{3}\right), 5.0$ (s, $2 \mathrm{H}, 2$ ', 5 ' -OH), $6.72(\mathrm{~d}, J=7.9 \mathrm{~Hz}, 2 \mathrm{H}, 3$ " , 5 " - $\mathrm{H}), 7.11-6.75(\mathrm{~m}, J=8.3 \mathrm{~Hz}, 3 \mathrm{H}, \mathrm{Ar}-$
H), 7.19 (d, $J=7.9 \mathrm{~Hz}, 2 \mathrm{H}, 2$ " , 6" $-\mathrm{H}), 7.56(\mathrm{~s}, 1 \mathrm{H},-\mathrm{CH}=\mathrm{CH}-), 7.90$ (s, 1H, $\mathrm{CH}=\mathrm{CH}-), \mathrm{IR}\left(\mathrm{KBr} / \mathrm{cm}^{-1}\right): 3244(-\mathrm{OH}), 1732,1716(-\mathrm{CO}), 1683(-\mathrm{CH}=\mathrm{CH}-)$, 1577,1540 (aromatic), 834 (p-disubstituted benzene)

4-(2-fluorophenyl)-1-[1-(2-hydroxyphenyl)-3-phenylallylidene] semicarbazide (4)

${ }^{1} \mathrm{H}-\mathrm{NMR}\left(\mathrm{\delta} / \mathrm{ppm}\right.$ in $\left.\mathrm{CDCl}_{3}\right)$ : $4.70(\mathrm{~s}, 1 \mathrm{H}, 2-\mathrm{OH}), 7.30-7.70(\mathrm{~m}, J=8.26 \mathrm{~Hz}$, $13 \mathrm{H}, \mathrm{Ar}-\mathrm{H}) 7.91(\mathrm{~s}, 1 \mathrm{H},-\mathrm{CH}=\mathrm{CH}-), 8.32(\mathrm{~s}, 1 \mathrm{H},-\mathrm{CH}=\mathrm{CH}-), 6.56(\mathrm{~s}, 1 \mathrm{H}$, ArNH, $\mathrm{D}_{2} \mathrm{O}$ exchangeable), $9.78\left(\mathrm{~s}, 1 \mathrm{H}, \mathrm{CONH}, \mathrm{D}_{2} \mathrm{O}\right.$ exchangeable); IR ( $\mathrm{KBr}$ $\left.\mathrm{cm}^{-1}\right)$ : $3431(\mathrm{NH}), 3478(-\mathrm{OH}), 3315-3235(\mathrm{CONH}), 1674(-\mathrm{CH}=\mathrm{CH}-), 1599$ (C-N), 1613, 1560 (aromatic), 755, 698 (monosubstituted benzene); MS, $\mathrm{m} / \mathrm{z}$ 374; Elemental analysis cal/fou (\%) C (70.39/70.32), H (4.83/4.68), N (11.19/10.98).

Synthesis of 2-flouro- phenyl urea (1)

2 -flouro aniline $(0.1 \mathrm{~mol})$ was dissolved in $20 \mathrm{ml}$ of glacial acetic acid and $10 \mathrm{ml}$ of water. To this, $0.1 \mathrm{~mol}$ of sodium cyanate $(6.5 \mathrm{~g})$ in $80 \mathrm{ml}$ of warm water was added with continuous stirring. The reaction mixture was allowed to stand for $30 \mathrm{~min}$ and then cooled in ice. The resultant crude solid was filtered with suction, dried and recrystallized with boiling water to yield 1. m.p $218^{\circ} \mathrm{C}$, ${ }^{1} \mathrm{H}-\mathrm{NMR}\left(\delta / \mathrm{ppm}\right.$ in $\left.\mathrm{CDCl}_{3}\right)$ : 7.12-7.64 (m, $\left.J=8.3 \mathrm{~Hz}, 3 \mathrm{H}, \mathrm{Ar}-\mathrm{H}\right), 8.02(\mathrm{~s}$, $1 \mathrm{H}$, imine- $\mathrm{H}), 8.34$ (s, $1 \mathrm{H}, \mathrm{ArNH}, \mathrm{D}_{2} \mathrm{O}$ exchangeable), $9.42(\mathrm{~s}, 2 \mathrm{H}, \mathrm{CONH}$, $\mathrm{D}_{2} \mathrm{O}$ exchangeable); IR $\left(\mathrm{KBr} / \mathrm{cm}^{-1}\right) 3260,3040,2850,1710,1600-1540,1240$. Synthesis of 2-flourophenyl semicarbazide(2)

Equimolar quantities $(0.05 \mathrm{~mol})$ of above fluorophenyl urea (1) $(9.2 \mathrm{~g})$ and hydrazine hydrate $(2.5 \mathrm{ml}, \mathrm{x} \mathrm{mmol})$ in ethanol were refluxed for $27 \mathrm{~h}$ with continuous stirring. The two-third volume of alcohol was distilled by vacuum distillation unit. Then the remaining reaction mixture was poured into ice. The resultant precipitate was filtered, washed with water and dried. The obtained solid was recrystallized with $50 \mathrm{ml}$ of $90 \%$ ethanol?. Mp $196^{\circ} \mathrm{C}{ }^{1} \mathrm{H}-\mathrm{NMR}(\delta /$ ppm in $\left.\mathrm{CDCl}_{3}\right): 5.46\left(\mathrm{~s}, 2 \mathrm{H}, \mathrm{NH}_{2}, \mathrm{D}_{2} \mathrm{O}\right.$ exchangeable), 7.32-7.84 (m, $J=8.38$ $\mathrm{Hz}, 3 \mathrm{H}, \mathrm{Ar}-\mathrm{H}), 8.32\left(\mathrm{~s}, 1 \mathrm{H}\right.$, imine-H), 8.34 (s, $1 \mathrm{H}, \mathrm{ArNH}, \mathrm{D}_{2} \mathrm{O}$ exchangeable), $9.86\left(\mathrm{~s}, 2 \mathrm{H}, \mathrm{CONH}, \mathrm{D}_{2} \mathrm{O}\right.$ exchangeable); IR $\left(\mathrm{KBr} / \mathrm{cm}^{-1}\right) 3245,3047,2837$, 1743, 1618-1537, 1241. (4-13)

Generalmethod for the synthesis of 2-flourophenyl chalconylsemicarbazone

To a solution of fluorophenyl semicarbazide (2) $(0.005 \mathrm{~mol}, 1.175 \mathrm{~g})$ in $25 \mathrm{ml}$ of ethanol added an equimolar quantity of the appropriate chalcone derivatives previously dissolved in $25 \mathrm{ml}$ of ethanol. Then few drops of concentrated hydrochloric acid were added. Continuous stirring was done for 4-5 hr, then reaction mixture poured on ice. Immediate precipitation was observed. The resultant precipitate was filtered, washed with sodium acetate $(0.005 \mathrm{~mol}, 0.41 \mathrm{~g})$ in $2 \mathrm{ml}$ water, dried and recrystallized with hot ethanol. The physicochemical parametes of the synthesized compounds were given in table 2 .

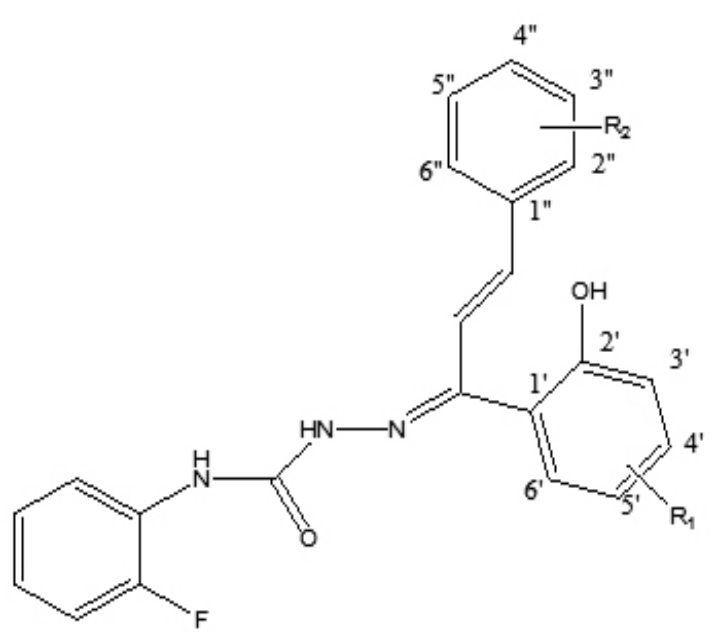


Table 2: Structure and physicochemical properties of synthesized title compounds.

\begin{tabular}{|c|c|c|c|c|c|}
\hline $\begin{array}{c}\text { Com } \\
\text { no }\end{array}$ & $\mathbf{R}_{1}$ & $\mathbf{R}_{2}$ & $\begin{array}{c}\text { Yield } \\
(\%)\end{array}$ & $\begin{array}{l}\text { Mp } \\
\left({ }^{\circ} \mathbf{C}\right)\end{array}$ & $\mathbf{R}_{\mathrm{f}}$ \\
\hline 4 & $\mathrm{H}$ & $\mathrm{H}$ & 59 & 122 & 0.77 \\
\hline 5 & $\mathrm{H}$ & 4"-OH & 65 & 127 & 0.70 \\
\hline 6 & $\mathrm{H}$ & 4"--OCH3 & 65 & 136 & 0.64 \\
\hline 7 & $\mathrm{H}$ & $4 "-\mathrm{N}(\mathrm{CH} 3) 2$ & 58 & 125 & 0.56 \\
\hline 8 & 4'-OH & 6"-OH & 65 & 115 & 0.62 \\
\hline 9 & $4^{\prime}-\mathrm{OH}$ & 4"-N(CH3)2 & 50 & 162 & 0.68 \\
\hline 10 & $\mathrm{H}$ & 6"-OH & 63 & 145 & 0.54 \\
\hline 11 & $5^{\prime} \mathrm{OH}$ & 6"-OH & 60 & 125 & 0.62 \\
\hline 12 & $5^{\prime} \mathrm{OH}$ & 4"-OH & 56 & 133 & 0.69 \\
\hline 13 & $5^{\prime} \mathrm{OH}$ & 4"-OCH3 & 57 & 162 & 0.52 \\
\hline
\end{tabular}

4-(2-fluorophenyl)-1-[1-(2-hydroxyphenyl)-3-(4-hydroxyphenyl) allylidene] semicarbazide (5)

${ }^{1} \mathrm{H}-\mathrm{NMR}\left(\delta / \mathrm{ppm}\right.$ in $\left.\mathrm{CDCl}_{3}\right): 4.75$ (s, $\left.1 \mathrm{H}, 4-\mathrm{OH}\right), 4.71(\mathrm{~s}, 1 \mathrm{H}, 2-\mathrm{OH})$, 7.25-7.50 (m, $J=8.2 \mathrm{~Hz}, 12 \mathrm{H}, \mathrm{Ar}-\mathrm{H}), 8.2(\mathrm{~s}, 1 \mathrm{H},-\mathrm{CH}=\mathrm{CH}-), 8.45(\mathrm{~s}, 1 \mathrm{H}$, $-\mathrm{CH}=\mathrm{CH}-), 6.55\left(\mathrm{~s}, 1 \mathrm{H}, \mathrm{ArNH}, \mathrm{D}_{2} \mathrm{O}\right.$ exchangeable), $9.68(\mathrm{~s}, 1 \mathrm{H}, \mathrm{CONH}$, $\mathrm{D}_{2} \mathrm{O}$ exchangeable); IR $\left(\mathrm{KBr} / \mathrm{cm}^{-1}\right): 3462(\mathrm{NH}), 3492(-\mathrm{OH}), 3313-3248$ (CONH), $1674(-\mathrm{CH}=\mathrm{CH}-), 1598(\mathrm{C}-\mathrm{N}), 1615,1556$ (aromatic), 753, 691 (monosubstituted benzene); MS, m/z 390; Elemental analysis cal/fou (\%) C (67.51/67.12), H (4.64/4.36), N (10.74/10.56).

4-(2-fluorophenyl)-1-[1-(2-hydroxyphenyl)-3-(4-methoxyphenyl) allylidene] semicarbazide (6)

${ }^{1} \mathrm{H}-\mathrm{NMR}\left(\delta / \mathrm{ppm}\right.$ in $\left.\mathrm{CDCl}_{3}\right): 4.72(\mathrm{~s}, 1 \mathrm{H}, 2-\mathrm{OH}), 3.89(\mathrm{~s}, 3 \mathrm{H}$, 4-OCH $\left.{ }_{3}\right), 7.38-7.59$ (m, $\left.J=8.2 \mathrm{~Hz}, 12 \mathrm{H}, \mathrm{Ar}-\mathrm{H}\right), 8.21$ (s, $\left.1 \mathrm{H},-\mathrm{CH}=\mathrm{CH}-\right)$, $8.365(\mathrm{~s}, 1 \mathrm{H},-\mathrm{CH}=\mathrm{CH}-), 6.45\left(\mathrm{~s}, 1 \mathrm{H}, \mathrm{ArNH}, \mathrm{D}_{2} \mathrm{O}\right.$ exchangeable), 9.55 (s, $1 \mathrm{H}, \mathrm{CONH}, \mathrm{D}_{2} \mathrm{O}$ exchangeable); IR $\left(\mathrm{KBr} / \mathrm{cm}^{-1}\right): 3452(\mathrm{NH}), 3482(-\mathrm{OH})$, 3305-3245 (CONH), 1677 ( $-\mathrm{CH}=\mathrm{CH}-), 1592(\mathrm{C}-\mathrm{N}), 1618,1568$ (aromatic), 755, 696 (monosubstituted benzene); MS, m/z 404; Elemental analysis (\%) C (68.14/67.96), H (4.97/4.68), N (10.36/10.02).

1-[1-(2,4-dihydroxyphenyl)-3-(2-hydroxyphenyl)allylidene]-4-(2fuorophenyl) semicarbazide (8)

${ }^{1} \mathrm{H}-\mathrm{NMR}\left(\delta / \mathrm{ppm}\right.$ in $\left.\mathrm{CDCl}_{3}\right): 5.2(\mathrm{~s}, 1 \mathrm{H}, 2-\mathrm{OH}), 5.0(\mathrm{~s}, 1 \mathrm{H}, 4-\mathrm{OH}), 5.4(\mathrm{~s}$, $1 \mathrm{H}, 6-\mathrm{OH}) \quad 7.20-7.70(\mathrm{~m}, J=8.34 \mathrm{~Hz}, 11 \mathrm{H}, \mathrm{Ar}-\mathrm{H}) 7.6(\mathrm{~s}, 1 \mathrm{H},-\mathrm{CH}=\mathrm{CH}-)$, 8.11 (s, $1 \mathrm{H},-\mathrm{CH}=\mathrm{CH}-), 6.53$ (s, $1 \mathrm{H}, \mathrm{ArNH}, \mathrm{D}_{2} \mathrm{O}$ exchangeable), $9.78(\mathrm{~s}, 1 \mathrm{H}$, CONH, $\mathrm{D}_{2} \mathrm{O}$ exchangeable); IR $\left(\mathrm{KBr} / \mathrm{cm}^{-1}\right): 3458(\mathrm{NH}), 3470(-\mathrm{OH}), 3325-$ $3260(\mathrm{CONH}), 1680(-\mathrm{CH}=\mathrm{CH}-), 1598(\mathrm{C}-\mathrm{N}), 1618,1568$ (aromatic), 755, 697 (monosubstituted benzene); MS, m/z 406; Elemental analysis cal/fou (\%) $\mathrm{C}(64.86 / 64.76), \mathrm{H}(4.45 / 4.26), \mathrm{N}(10.31 / 10.22)$.

1-[1-(2,5-dihydroxyphenyl)-3-(2-hydroxyphenyl)allylidene]-4-(2fluorophenyl) semicarbazide (11)

${ }^{1} \mathrm{H}-\mathrm{NMR}\left(\delta / \mathrm{ppm}\right.$ in $\left.\mathrm{CDCl}_{3}\right): 5.31(\mathrm{~s}, 1 \mathrm{H}, 2-\mathrm{OH}), 4.9(\mathrm{~s}, 1 \mathrm{H}, 4-\mathrm{OH}), 5.6$ (s, $1 \mathrm{H}, 6-\mathrm{OH}) \quad 7.20-7.74(\mathrm{~m}, J=8.4 \mathrm{~Hz}, 11 \mathrm{H}, \mathrm{Ar}-\mathrm{H}) 7.86(\mathrm{~s}, 1 \mathrm{H},-\mathrm{CH}=\mathrm{CH}-)$, $8.17(\mathrm{~s}, 1 \mathrm{H},-\mathrm{CH}=\mathrm{CH}-), 6.47$ (s, $1 \mathrm{H}, \mathrm{ArNH}, \mathrm{D} 2 \mathrm{O}$ exchangeable), $9.60(\mathrm{~s}, 1 \mathrm{H}$, $\mathrm{CONH}, \mathrm{D}_{2} \mathrm{O}$ exchangeable); IR $\left(\mathrm{KBr} / \mathrm{cm}^{-1}\right): 3465(\mathrm{NH}), 3475(-\mathrm{OH}), 3310-$ $3250(\mathrm{CONH}), 1677(-\mathrm{CH}=\mathrm{CH}-), 1595(\mathrm{C}-\mathrm{N}), 1619,1557$ (aromatic), 754, 690 (monosubstituted benzene); MS, m/z 406; Elemental analysis cal/fou (\%) C (64.86/64.56), H (4.45/4.14), N (10.31/9.98).

1-[1-(2,5-dihydroxyphenyl)-3-(4-methoxyphenyl)allylidene]-4-(2fluorophenyl) semicarbazide (13)

${ }^{1} \mathrm{H}-\mathrm{NMR}\left(\delta / \mathrm{ppm}\right.$ in $\left.\mathrm{CDCl}_{3}\right): 3.77$ (s, 3H, 4-OCH $), 5.3(\mathrm{~s}, 1 \mathrm{H}, 2-\mathrm{OH}), 5.64$ $(\mathrm{s}, 1 \mathrm{H}, 5-\mathrm{OH}), 7.15-7.75(\mathrm{~m}, J=8.3 \mathrm{~Hz}, 11 \mathrm{H}, \mathrm{Ar}-\mathrm{H}), 7.82(\mathrm{~s}, 1 \mathrm{H},-\mathrm{CH}=\mathrm{CH}-)$, $7.94(\mathrm{~s}, 1 \mathrm{H},-\mathrm{CH}=\mathrm{CH}-), 6.70\left(\mathrm{~s}, 1 \mathrm{H}, \mathrm{ArNH}, \mathrm{D}_{2} \mathrm{O}\right.$ exchangeable), $9.98(\mathrm{~s}, 1 \mathrm{H}$, CONH, $\mathrm{D}_{2} \mathrm{O}$ exchangeable); IR $\left(\mathrm{KBr} / \mathrm{cm}^{-1}\right): 3453(\mathrm{NH}), 3483(-\mathrm{OH}), 3308-$ $3238(\mathrm{CONH}), 1685(-\mathrm{CH}=\mathrm{CH}-), 1594(\mathrm{C}-\mathrm{N}), 1614,1547$ (aromatic), 756, 687 (monosubstituted benzene); MS, m/z 420; Elemental analysis cal/fou (\%) C (65.55/65.24), H (4.78/4.48), N (9.97/9.72).

Pharmacological Screening

The anticonvulsant evaluations were undertaken using reported procedures $[17,18]$. Initially all the compounds were administered i.p. at doses of 100 and $300 \mathrm{mg} / \mathrm{kg}$ to one to four animals. Activity was established using the MES, scPTZ and neurotoxicity models and these data are presented in Table 3.
Behavioral testing

The title compounds ( $30 \mathrm{mg} / \mathrm{kg}$ ) were screened for their behavioral effects $[19,20]$ using actophotometer at $30 \mathrm{~min}$ and $1 \mathrm{~h}$ after injection. The behavior of animals inside the photocell was recorded as a digital score. The control animal was administered DMSO. The observations are tabulated as Table 4 .

Table 3: pharmacological screening as anticonvulsant of synthesized compounds.

\begin{tabular}{|c|c|c|c|c|c|c|}
\hline \multirow{2}{*}{ comp } & \multicolumn{3}{c|}{ Intraperitonial Injection $^{\mathbf{a}}$} \\
\cline { 2 - 7 } & \multicolumn{2}{|c|}{ MES screen } & \multicolumn{2}{c|}{ ScPTZ screening } & \multicolumn{2}{c|}{ Toxicity screen } \\
\hline & $\mathbf{0 . 5 h}$ & $\mathbf{4 h}$ & $\mathbf{0 . 5 h}$ & $\mathbf{4 h}$ & $\mathbf{0 . 5 h}$ & $\mathbf{4 h}$ \\
\hline $\mathbf{4}$ & 300 & - & 300 & - & - & - \\
\hline $\mathbf{5}$ & 100 & 300 & 300 & - & 300 & - \\
\hline $\mathbf{6}$ & 300 & - & 300 & - & 300 & - \\
\hline $\mathbf{7}$ & 300 & - & 100 & 300 & 300 & - \\
\hline $\mathbf{8}$ & 100 & 300 & 100 & 300 & 100 & 300 \\
\hline $\mathbf{9}$ & 100 & 300 & 300 & - & 300 & - \\
\hline $\mathbf{1 0}$ & 100 & 300 & 300 & - & 300 & - \\
\hline $\mathbf{1 1}$ & 100 & 300 & 100 & 300 & 100 & 300 \\
\hline $\mathbf{1 2}$ & 100 & 300 & 100 & 300 & 300 & - \\
\hline $\mathbf{1 3}$ & 100 & 300 & 300 & - & 300 & - \\
\hline
\end{tabular}

a Doses of 30,100 and $300 \mathrm{mg} / \mathrm{kg}$ were administered. The figures in the table indicate the minimum dose whereby bioactivity was demonstrated in half or more of the mice. The dash (-) indicates an absence of activity at maximum dose administered $(300 \mathrm{mg} / \mathrm{kg})$.

Table 4: Behavioral study on synthesized compounds using actophotometer.

\begin{tabular}{|c|c|c|c|}
\hline \multirow{2}{*}{ Compound $^{\text {a }}$} & \multirow{2}{*}{$\begin{array}{c}\text { Activity score } \\
\text { Control (24 h } \\
\text { prior) }\end{array}$} & \multicolumn{2}{|c|}{ Post-treatment } \\
\cline { 3 - 4 } & & $\mathbf{0 . 5}$ h after & 1 h after \\
\hline $\mathbf{4}$ & $320 \pm 27.73$ & $531 \pm 17.22$ & $343 \pm 21.11$ \\
\hline $\mathbf{5}$ & $180 \pm 30.22$ & $161 \pm 9.69^{*}$ & $164 \pm 11.02^{*}$ \\
\hline $\mathbf{6}$ & $303 \pm 22.66$ & $241 \pm 2.21^{* *}$ & $247 \pm 1.02^{*}$ \\
\hline $\mathbf{7}$ & $519 \pm 13.38$ & $421 \pm 26.78^{*}$ & $459 \pm 29.87^{*}$ \\
\hline $\mathbf{8}$ & $373 \pm 32.75$ & $20 \pm 6.12^{*}$ & $22 \pm 2.35^{*}$ \\
\hline $\mathbf{9}$ & $320 \pm 27.73$ & $231 \pm 17.22$ & $243 \pm 21.11$ \\
\hline $\mathbf{1 0}$ & $408 \pm 32.96$ & $248 \pm 9.66^{*}$ & $333 \pm 2.11^{*}$ \\
\hline $\mathbf{1 1}$ & $247 \pm 15.66$ & $55 \pm 7.25^{*}$ & $57 \pm 7.35^{*}$ \\
\hline $\mathbf{1 2}$ & $320 \pm 27.73$ & $31 \pm 17.22$ & $43 \pm 21.11$ \\
\hline $\mathbf{1 3}$ & $180 \pm 30.22$ & $110 \pm 9.69^{*}$ & $164 \pm 11.02^{*}$ \\
\hline Phenytoin $^{\mathbf{c}}$ & $267 \pm 31.12$ & $44 \pm 4.56^{* *}$ & $46 \pm 2.44^{* *}$ \\
\hline
\end{tabular}

${ }^{\text {a }}$ The compounds were tested at a dose of $30 \mathrm{mg} / \mathrm{kg}$ (i.p.).

${ }^{b}$ Each score represents the mean \pm SEM of six mice, significantly different from the control score,

$* P<0.05, * * P<0.01$ (Dunnett's post hoc test)

${ }^{c}$ Tested at $5 \mathrm{mg} / \mathrm{kg}$ p.o. 
Table: 5 CNS study on selected compounds in forced swim pool test.

\begin{tabular}{|c|c|c|}
\hline \multirow[t]{2}{*}{ Compa } & \multicolumn{2}{|c|}{ Immobility time $^{b}$} \\
\hline & $\begin{array}{l}\text { Control (24 hrs } \\
\text { prior) }\end{array}$ & $\begin{array}{c}\text { Post- treatment (60 min } \\
\text { after) }\end{array}$ \\
\hline DMSO & $158.67 \pm 11.68$ & $168.53 \pm 12.32$ \\
\hline 4 & $128.67 \pm 11.56$ & $180.30 \pm 12.45^{*}$ \\
\hline 5 & $119 \pm 12.35$ & $130.3 \pm 11.25^{*}$ \\
\hline 6 & $142 \pm 11.70$ & $168.00 \pm 11.73$ \\
\hline 7 & $125.67 \pm 11.90$ & $155.30 \pm 12.35^{*}$ \\
\hline 8 & $57 \pm 12.16$ & $130.3 \pm 11.26^{* *}$ \\
\hline 9 & $124 \pm 12.70$ & $183.00 \pm 11.64$ \\
\hline 10 & $88.65 \pm 12.62$ & $104.00 \pm 12.94 *$ \\
\hline 11 & $85.25 \pm 11.63$ & $167.30 \pm 12.49$ \\
\hline 12 & $75.67 \pm 12.05$ & $150.60 \pm 12.06 *$ \\
\hline 13 & $124.33 \pm 13.29$ & $187.3 \pm 13.09$ \\
\hline carbamazepine & $138.4 \pm 17.3$ & $240.60 \pm 14.10^{*}$ \\
\hline
\end{tabular}

${ }^{a}$ The compounds were tested at a dose of $30 \mathrm{mg} / \mathrm{kg}$ (i.p.).

${ }^{\mathrm{b}}$ Each value represents the mean \pm SEM of six rats significantly different from the control,

$* P<0.05$ and $* * P<0.01$ (Dunnett's post hoc test).

\section{RESULTS AND DISCUSSION}

The synthesized title compounds (4-13) obtained from the reaction sequence were injected intraperitoneally into mice and evaluated in the maximal electroshock (MES), subcutaneous pentylenetetrazole (scPTZ) and neurotoxicity screens, using doses of 30,100 , and $300 \mathrm{mg} / \mathrm{kg}$, and observation carried out at two different time intervals $(0.5$ and $4 \mathrm{~h})$. These data are presented in Table 3. All the compounds showed anti-MES activity indicative of their ability to prevent seizure spread. Compounds that showed protection against MES model at $100 \mathrm{mg} / \mathrm{kg}$ were include 5, 8, 9, 10, 11, 12 and 13. The compounds $5,8,9,10,11,12$ and 13 were shown activity both at $0.5 \mathrm{~h}$ and $4.0 \mathrm{~h}$ periods. Compound $\mathbf{4 , 6}$ and 7 were shown activity only at $0.5 \mathrm{~h}$ at the dose of $300 \mathrm{mg} / \mathrm{kg}$, indicating that they have rapid onset and shorter duration of action. Most of the compounds were found to be active in the scPTZ test, a test used for identify compounds that elevate seizure threshold. Compounds 7, 8, 11 and 12 were shown activity at a dose of $100 \mathrm{mg} / \mathrm{kg}$. All these compounds were shown $100 \%$ protection at a dose of $300 \mathrm{mg} / \mathrm{kg}$ at $0.5 \mathrm{~h}$. So these compounds were having quick onset of action but for shorter duration. In the neurotoxicity screen, compounds $\mathbf{8}$ and $\mathbf{1 1}$ were not shown neurotoxicity in the maximum administered dose $(300 \mathrm{mg} / \mathrm{kg})$. On the other hand, most of the synthesized compounds were neurotoxic at the maximum administered dose (300 mg/kg). Among the synthesized compounds, the unsubstituted derivative 4 exhibited activity against MES and scPTZ models. When the phenyl group of aldehydic and acetophenic moiety of chalcone was substituted with-OH group (compound 8, 10 and 12) the compounds exhibited activity in all model in comparison to substituted with other as methoxy and p-dimethyl amino groups (compound 6, 7,9 and 13) are active in more than one model. All compounds were studied for the CNS behavioral activity in mice using actophotometer and forced swimming pool test models the results are presented in Table 4 and 5 . In the behavioral study, using actophotometer, the compounds $\mathbf{4}$ showed no behavioral despair effect after $1.0 \mathrm{~h}$ when compared to Phenytoin. All other compounds were found to decrease the activity of the animals.

\section{ACKNOWLEDGEMENTS}

The authors deeply appreciate the assistance of the Antiepileptic Drug Development program, Epilepsy Branch, Preclinical Pharmacology Section, $\mathrm{NIH}$, USA in the testing of the compounds.

\section{REFERENCES}

[1] D. McCormick, D. Contreras, Annu. Rev. Physiol. 63, 815, (2001).

[2] O. J. McNamara, Pharmacological Basis of Therapeutics; Hardman J. G., Limbird L. E., Gilman A. G., Eds.; McGraw-Hill: New York, 521, (2001).

[3] K. J. Meador, J. Clin. Psychiatry. 64 (Suppl. 8), 30, (2003).

[4] Z. Lin, P. K. Kadaba, Med. Res. Rev. 17, 537, (1997).

[5] P. Yogeeswari, J. Raghavendran, R. Thirumurugan, A. Saxena, D. Sriram, Curr. Drug Targets. 5, 553, (2004).

[6] P. Yogeeswari, D. Sriram, V. Saraswat, J. Ragavendran, M. Kumar, M. Murugesan, R. Thirumurugan, J. P. Stables, Eur. J. Pharm. Sci. 20, 341, (2003).

[7] S. N. Pandeya, I. Ponnilavarasan, A. Pandey, R. Lakhan, J. P. Stables, Pharmazie. 54, 923, (1999).

[8] J. R. Dimmock, S. C. Vashishta, J. P. Stables, Eur. J. Med. Chem. 35, 241, (2000).

[9] J. R. Dimmock, G. B. Baker, Epilepsia. 35, 648, (1994).

[10] J. R. Dimmock, S. C. Vashishta, J. P. Stables, Pharmazie. 55, 490, (2000).

[11] S. N. Pandeya, P. Yogeeswari, J. P. Stables, Eur. J. Med. Chem. 35, 879, (2000).

[12] S. N. Pandeya, V. Mishra, I. Ponnilavarasan, J. P. Stables, Pol. J. Pharmacol. 52, 283, (2000).

[13] S. N. Pandeya, H. Manjula, J. P. Stables, Pharmazie. 56, 121, (2001).

[14] J. R. Dimmock, S. S. Jonnalagadda, S. Hussain, S. Tiwari, J. W. Quail, R. S. Reid, L. T. Delbaere, L. Prasad, Eur. J. Med. Chem. 25, 581, (1990).

[15] P. Yogeeswari, D. Sriram, R. Thirumurugan, J. Raghavendran, J. Med. Chem. 48, 6202, (2005).

[16] [P. Yogeeswari, D. Sriram, V. Saraswat, J. Ragavendran, M. Kumar, S. Murugesan R. Thirumurugan, J. P. Stables, Eur. J. Pharm. Sci. 20, 341, (2003).

[17] R. L. Krall, J. K. Penry, B. G. White, H. Kupferbeng, Epilepsia. 19, 409, (1978).

[18] R. J. Porter, J. J. Cereghino, G. D.Gladding, B. Hessie, H. J. Kupferberg, B. Scoville, B. White, Cleveland Clin. Q. 51, 293, (1984).

[19] J. R. Boissier, P. Simon, Arch. Int. Pharmacodyn. Ther. 158, 212, (1965).

[20] R. D. Porsolt, G. Anton, N. Blanet, M. Jalbre, Eur. J. Pharmacol. 47, 379, (1978). 